\title{
Effects of high-frequency transcranial magnetic stimulation on functional performance in individuals with incomplete spinal cord injury: study protocol for a randomized controlled trial
}

\author{
Amanda Vitória Lacerda de Araújo', Valéria Ribeiro Nogueira Barbosa², Gilma Serra Galdino², Felipe Fregni ${ }^{3}$, \\ Thais Massetti ${ }^{1}$, Sara Lynn Fontes ${ }^{4}$, Danilo de Oliveira Silva ${ }^{5}$, Talita Dias da Silva', \\ Carlos Bandeira de Mello Monteiro ${ }^{1}$, James Tonks ${ }^{6,7,8}$ and Fernando Henrique Magalhães, ${ }^{1,9^{*}}$
}

\begin{abstract}
Background: Repetitive transcranial magnetic stimulation (rTMS) has been investigated as a new tool in neurological rehabilitation of individuals with spinal cord injury (SCI). However, due to the inconsistent results regarding the effects of rTMS in people with $\mathrm{SCl}$, a randomized controlled double-blind crossover trial is needed to clarify the clinical utility and to assess the effect size of rTMS intervention in this population. Therefore, this paper describes a study protocol designed to investigate whether the use of rTMS can improve the motor and sensory function, as well as reduce spasticity in patients with incomplete $\mathrm{SCl}$.

Methods: A double-blind randomized sham-controlled crossover trial will be performed by enrolling 20 individuals with incomplete SCl. Patients who are at least six months post incomplete SCl (aged 18-60 years) will be recruited through referral by medical practitioners or therapists. Individuals will be randomly assigned to either group 1 or group 2 in a 1:1 ratio, with ten individuals in each group. The rTMS protocol will include ten sessions of high-frequency rTMS $(5 \mathrm{~Hz})$ over the bilateral lower-limb motor area positioned at the vertex $(\mathrm{Cz})$. Clinical evaluations will be performed at baseline and after rTMS active and sham.
\end{abstract}

Discussion: rTMS has produced positive results in treating individuals with physical impairments; thus, it might be promising in the SCl population. The results of this study may provide new insights to motor rehabilitation thereby contributing towards the better usage of rTMS in the SCl population.

Trial registration: ClinicalTrials.gov, NCT02899637. Registered on 25 August 2016.

Keywords: Incomplete spinal cord injury, Plasticity, Motor rehabilitation, Non-invasive brain stimulation, Repetitive transcranial magnetic stimulation

\footnotetext{
* Correspondence: fhmagalhaes@usp.br; fhmagalhaess@gmail.com

${ }^{1}$ School of Arts, Sciences and Humanities, EACH - USP, University of São Paulo, São Paulo, Brazil

${ }^{9}$ Biomedical Engineering Laboratory, Department of Telecommunication and

Control, Avenida Professor Luciano Gualberto, Travessa 3, n. 158. Cidade

Universitária, São Paulo, SP 05508-010, Brazil

Full list of author information is available at the end of the article
} 


\section{Background}

Spinal cord injury (SCI) affects about 2.5 million individuals worldwide and often leads to severe disability, due to functional limitations in the sensory and motor systems [1]. After trauma or pathological processes upon the spinal cord, there is usually some preservation of sensation, or motor function at the lowest segment of the spinal cord, a condition known as incomplete SCI (iSCI) [2]. iSCI has been associated with a serious reduction in quality of life and functional independence [3, 4]. Therefore, effective rehabilitation programs are required for patients with iSCI in both acute and chronic care.

Processes of neural regeneration and plasticity can result in significant functional recovery after iSCI [5]. Spontaneous recovery of motor and sensory function in iSCI individuals can be substantial, but highly variable [6]. Repetitive transcranial magnetic stimulation (rTMS) rehabilitation techniques, based on protocols that selectively stimulate specific pathways along the central nervous system (CNS), have been found to be effective in enhancing neurological recovery leading to improved functional abilities [7]. rTMS is a procedure that involves repetitively delivering biphasic magnetic pulses over a specific cortical site $[8,9]$ to provide stimulation of the corticospinal tract (CST), primary motor cortex (M1), and spinal cord, so as to induce neuronal reorganization, which can be largely involved in the control of voluntary movements [10].

Protocols involving rTMS have been used to induce changes in the excitability of neuronal circuits with positive effects [11-16] at the site of stimulation or transsynaptically at distant sites such as spinal cord circuits $[17,18]$. The effect of high-frequency rTMS (i.e. $\geq 5 \mathrm{~Hz}$ ) includes changes in synaptic plasticity resembling long-term potentiation (LTP), as well as shifts in network excitability, activation of feedback loops, and activitydependent metaplasticity $[19,20]$. On this basis, rTMS is emerging as a promising technique in improving neurophysiological outcomes and voluntary motor output in patients with motor disorders [11, 21, 22]. Indeed, studies indicate that motor improvements may be due to modification of corticospinal projections by increasing motor cortical excitability [14-16] and, consequently, promote plasticity associated with functional recovery [22]. Additionally, reduction in spasticity may occur through enhancement of descending corticospinal projections and segmental effects on spinal interneurons that might strengthen inhibitory connections $[13,14,23]$.

Many studies have used rTMS as a non-invasive and painless method to induce long-lasting changes in the excitability of cortical and corticospinal pathways after iSCI. In this scenario, rTMS has been found effective in enhancing corticospinal synaptic transmission [12], reducing spasticity $[13,14,23]$ and improving sensorimotor function after iSCI [14-16], although we note none of these studies reported associated effect sizes in their analyses. However, other studies report less positive findings, including unchanged sensorimotor function [24], as well as unaltered cortical excitability and level of spasticity [16] in response to rTMS. The lack of consistent results is likely associated with differences in stimulation parameters (e.g. intensity, frequency, number of pulses), number of sessions, relative location of M1, chronicity and levels of injury, and outcome measurements used in previous studies [10].

In considering three particular studies that have examined the effects of rTMS in individuals with iSCI, we note interesting results on sensory and motor performance. Belci et al. [15] delivered double pulses of rTMS over the M1 representation of thenar muscles (360 doublets, separated by $100 \mathrm{~ms}$ ). They used a low frequency (with $10 \mathrm{~s}$ between each doublet and stimulus intensity corresponding to $90 \%$ of resting motor threshold [RMT]) for five days in a group of four individuals with iSCI. Results indicated that active rTMS improved somatosensory and motor function at three weeks following stimulation, as assessed by the American Spinal Injury Association Impairment Scale (ASIA) nine-hole peg test and electrical perceptual threshold measurements. Additionally, Benito et al. [14] applied 15 sessions of sham or rTMS over the leg representation of M1 (20 trains of 40 pulses at $20 \mathrm{~Hz}$ with an intensity of $90 \%$ of RMT) in 17 individuals with iSCI. This study found significant improvements in motor function for at least two weeks, as assessed by the ASIA scale for lower limbs and gait function. Contrary to the findings noted above, ASIA scores were unaltered in Kuppuswamy et al. [24]. The protocol had five days of active or sham rTMS ( 900 pulses at $5 \mathrm{~Hz}$ in $2 \mathrm{~s}$ trains separated by $80-\mathrm{s}$ intervals, at $80 \%$ of active motor threshold) applied to M1 representations of either the hand or forearm muscles in 15 individuals with iSCI. It is worth noting that the studies cited above have reported sham placebo-controlled trials which were single-blinded [15, 24], non-randomized [15], and either all $[15,24]$, or at least part of the individuals crossed over between groups during the study [14].

Therefore, while there is good evidence for its use in other diseases, the literature regarding the efficacy of rTMS in individuals with iSCI is inconsistent, as the few studies that have addressed this issue yielded contrasting results. Due to the quality of the study design as well as to the differences in rTMS parameters used in previous studies, there is no strong evidence of consistent changes in sensory and motor function in individuals with iSCI after rTMS [10]. Thus, there are gaps in the knowledge base and hence the development of a new prospective study, with a fully randomized double-blind placebo-controlled design [25], is necessary to provide 
evidence of the clinical utility of rTMS in individuals with iSCI. Due to the positive effects of rTMS in inducing long-lasting changes in spinal and supraspinal circuits, we hypothesized that rTMS applied over the lower-limb motor area will: (1) improve motor function; (2) improve sensory function; and (3) reduce spasticity.

Therefore, our aim was to identify a protocol for a double-blind randomized controlled crossover trial that will be performed to evaluate the effects of highfrequency rTMS on the sensorimotor function and spasticity in individuals with iSCI.

\section{Methods/Design}

We registered this trial on ClinicalTrials.gov (NCT02899637). This paper has been reported in accordance with the Standard Protocol Items: Recommendations for Interventional Trials (SPIRIT) [26].

\section{Study design}

A double-blinded randomized controlled crossover trial will be conducted and all participants will undertake rTMS-active and sham. Group 1 will start with five sessions of rTMS-active. After two weeks, this group will be reallocated to five sessions with rTMS-sham. In contrast, group 2 will do the opposite protocol (participants will start allocated to five sessions of rTMS-sham, and after a two-week washout period will be reallocated to five sessions of rTMS-active). The two-week washout period has been used in some studies [24, 27] and was shown to be enough to reset the effects of the first five sessions, considering that the motor effects of the rTMS in individuals with iSCI are sustained for $1 \mathrm{~h}$ [24] to two weeks [14] and that spasticity effects endure for one week [16, 23]. Figure 1 summarizes the planned experimental design. This research protocol follows the SPIRIT recommendations. For the SPIRIT Checklist see Additional file 1 and for the SPIRIT Figure see Fig. 2. Table 1 provides an overview of the trial characteristics, based on the WHO Trial Registration Data Set.

\section{Participants and sampling}

Participants will be recruited through referral by medical practitioners or therapists who work at the Physiotherapy Health Center of Paraíba State University in Brazil in single-stage cluster sampling. Those interested in participating will undergo a detailed screening against the eligibility criteria for enrollment in the study.

\section{Inclusion criteria}

Participants will be included if they have agreed to participate in the study and have signed an informed consent form. They will have a clinical diagnosis of iSCI with nonprogressive etiology which is characterized by spinal, vascular, and infectious trauma. Neuroimaging examinations,

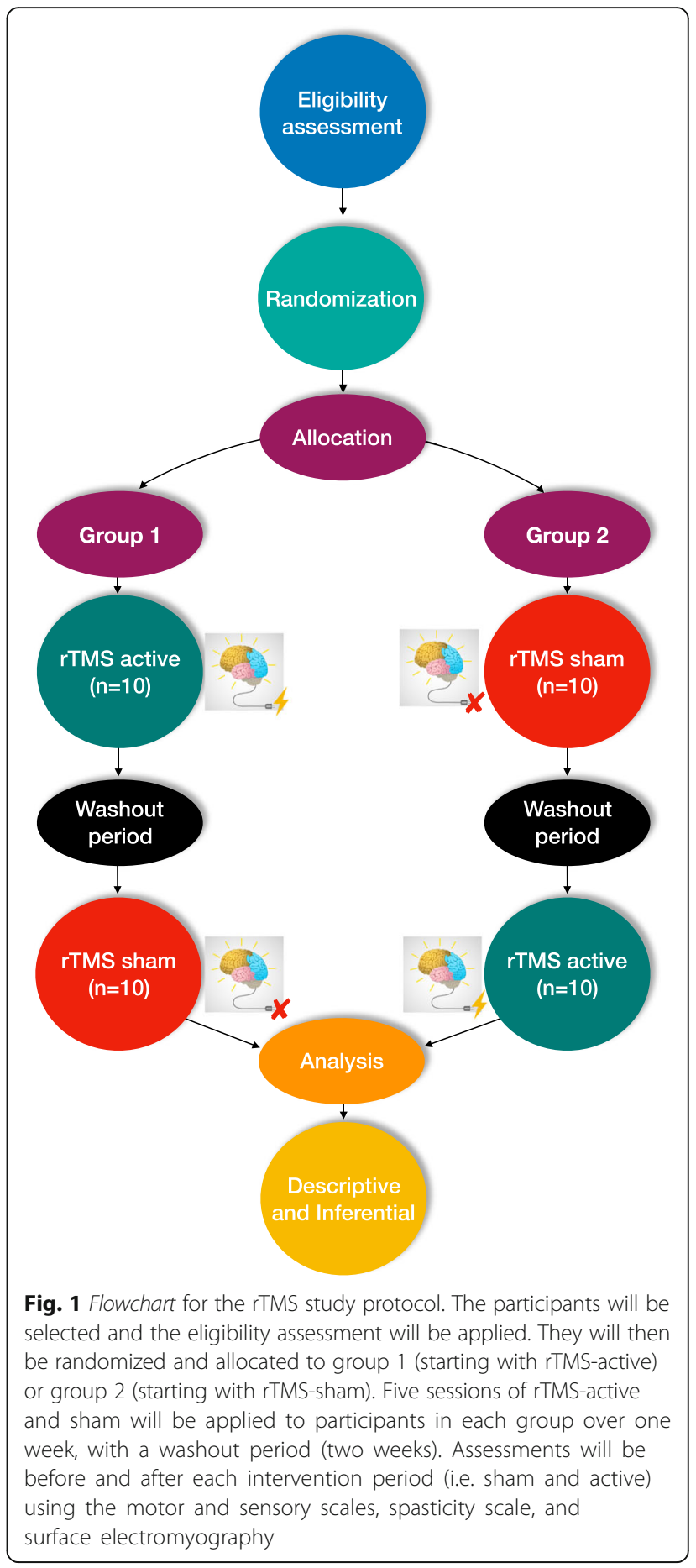

such as computed tomography and nuclear magnetic resonance imaging, will be used to exclude SCIs due to progressive worsening conditions such as neurodegenerative, tumor, and demyelinating pathologies $[28,29]$. The participants should be at least six months post iSCI, clinically stable, aged 18-60 years, have a satisfactory score in Scale of Mini-Mental State Examination (MMSE) (i.e. cut-off points of 13 for illiterates, 18 for low and middle school, 


\begin{tabular}{|c|c|c|c|c|c|c|c|}
\hline \multirow[b]{3}{*}{ TIMEPOINT** } & \multicolumn{7}{|c|}{ STUDY PERIOD } \\
\hline & \multirow{2}{*}{$\begin{array}{c}\text { Enrolment } \\
-t_{1}\end{array}$} & \multirow{2}{*}{$\begin{array}{c}\text { Allocation } \\
0\end{array}$} & \multicolumn{4}{|c|}{ Post-allocation } & \multirow{2}{*}{$\begin{array}{c}\begin{array}{c}\text { Close- } \\
\text { out }\end{array} \\
\begin{array}{c}\boldsymbol{t}_{5} \\
\text { week } 5\end{array} \\
\end{array}$} \\
\hline & & & $\begin{array}{c}t_{1} \\
\text { week } 1 \\
\end{array}$ & $\begin{array}{c}t_{2} \\
\text { week 2 }\end{array}$ & $\begin{array}{c}t_{3} \\
\text { week } 3 \\
\end{array}$ & $\begin{array}{c}t_{4} \\
\text { week } 4 \\
\end{array}$ & \\
\hline \multicolumn{8}{|l|}{ ENROLMENT: } \\
\hline \multirow{4}{*}{$\begin{array}{r}\text { Eligibility screen } \\
\text { Informed consent } \\
E E G \\
H A M-D \\
M M S E \\
S D Q\end{array}$} & $x$ & & & & & & \\
\hline & $\mathrm{x}$ & & & & & & \\
\hline & $x$ & & & & & & \\
\hline & & $x$ & & & & & \\
\hline \multicolumn{8}{|l|}{ INTERVENTIONS: } \\
\hline \multirow[t]{2}{*}{ Active rTMS } & & & $\mathrm{x}$ & \multicolumn{2}{|c|}{ Washout } & $\mathrm{X}$ & \\
\hline & & & $\mathrm{X}$ & \multicolumn{2}{|c|}{ Washout } & $\mathrm{X}$ & \\
\hline \multicolumn{8}{|l|}{ ASSESSMENTS: } \\
\hline \multirow{3}{*}{$\begin{array}{r}\text { ASIA motor } \\
\text { ASIA sensory } \\
F M S \\
E M G \\
M A S \\
N E \\
\text { ASIA motor } \\
\text { ASIA sensory } \\
F M S \\
E M G \\
M A S\end{array}$} & $x$ & & & & & & \\
\hline & & & & $\mathrm{x}$ & $x$ & & $\mathrm{x}$ \\
\hline & & & $x$ & $x$ & $x$ & $x$ & $\mathrm{x}$ \\
\hline
\end{tabular}

Fig. 2 SPIRIT figure. Description of the rTMS study protocol

and 26 for high school), have no pathological alterations on electroencephalography (EEG), be clear of depression as assessed by the Hamilton Depression Scale (HAM-D), and be currently receiving conventional sensorimotor physiotherapy, to a maximum of three times per week.

\section{Exclusion criteria}

Participants will be excluded if they have a metal prosthesis in some part of the body, use a cardiac pacemaker, have either cognitive impairment, psychotic, or schizophrenic disorders as diagnosed by the rehabilitation team, neuropsychiatric co-morbidity, or use drugs that reduce either seizure threshold or spasticity. Individuals participating in intensive programs of rehabilitation that could bias the results will also be excluded.

EG before the rTMS procedure will identify possible brain bioelectrical abnormalities (such as interictal epileptiform discharge, generalized photosensitivity, burst suppression, hypsarrhythmia) that could be related to the possibility of epileptic discharges $[30,31]$, thus reducing the risk of seizures during rTMS, since the high-frequency stimulation might lead to cortical hyperactivity. If any of these abnormalities are identified, the individual will be excluded.

\section{Dropout criteria}

Participants will be withdrawn from the study if they are not willing to continue their participation, cannot be present on the day of the experiment or miss a treatment section, and/or change their form of rehabilitation during the study.

\section{Randomization}

Participants will be randomly allocated to either group 1 (rTMS-active) or group 2 (rTMS-sham) with a 1:1 allocation defined by a computer-generated randomization using the $\mathrm{R}$ package ( $\mathrm{R}$ Foundation for Statistical Computing) [32]. Randomization will be under the control of a blinded investigator who will be the only person allowed to manage the electronic security file of the randomization to assign the individuals. The investigator will be blind to the group in which the participant is allocated to.

\section{Blinding}

The participants, researchers, and outcome assessors will remain blind to group allocation during the study. To ensure proper blinding, participants will receive 
Table 1 Trial characteristics based on WHO Trial Registration Data Set

\begin{tabular}{|c|c|}
\hline Data category & Trial information \\
\hline $\begin{array}{l}\text { Primary registry and trial } \\
\text { identifying number }\end{array}$ & ClinicalTrials.gov, ID: NCT02899637 \\
\hline $\begin{array}{l}\text { Date of registration in } \\
\text { primary registry }\end{array}$ & 25 August 2016 on \\
\hline $\begin{array}{l}\text { Secondary identifying } \\
\text { numbers }\end{array}$ & Ethical Committee of Paraiba State University, under the number CAEE: 18753713.0.0000.5187 \\
\hline $\begin{array}{l}\text { Source(s) of monetary or } \\
\text { material support }\end{array}$ & $\begin{array}{l}\text { Foundation of Support for Research of São Paulo } \\
\text { State - FAPESP \#2015/13096-1 and Coordination for Higher Education Staff Development - CAPES. }\end{array}$ \\
\hline Primary sponsor & University of Sao Paulo - USP \\
\hline Secondary sponsor(s) & NA \\
\hline $\begin{array}{l}\text { Contact for public } \\
\text { queries }\end{array}$ & FHM, AVLA \\
\hline $\begin{array}{l}\text { Contact for scientific } \\
\text { queries }\end{array}$ & FHM, AVLA \\
\hline Public title & Effects of transcranial magnetic stimulation on incomplete spinal cord injury \\
\hline Scientific title & $\begin{array}{l}\text { Effects of high-frequency transcranial magnetic stimulation on functional performance in individuals with incomplete spinal cord } \\
\text { injury: study protocol for a randomized controlled trial }\end{array}$ \\
\hline Country of recruitment & Brazil \\
\hline $\begin{array}{l}\text { Health condition(s) or } \\
\text { problem(s) studied }\end{array}$ & Spinal cord injury \\
\hline Interventions & $\begin{array}{l}\text { High-frequency repetitive transcranial magnetic stimulation on the lower-limb area of the motor cortex, over one week } \\
\text { (five consecutive sessions - once a day) }\end{array}$ \\
\hline $\begin{array}{l}\text { Key inclusion and } \\
\text { exclusion criteria }\end{array}$ & $\begin{array}{l}\text { Inclusion criteria: clinical diagnosis of iSCI with non-progressive etiology; at least six months post iSCl; clinical stability; age } \\
\text { range } 18-60 \text { years; satisfactory score in Scale of Mini-Mental State Examination (i.e. cut-off points of } 13 \text { for illiterates, } 18 \text { for low } \\
\text { and middle school, and } 26 \text { for high school); no pathological alterations on electroencephalography; absence of depression } \\
\text { as assessed by the Hamilton Depression Scale and receive sensorimotor conventional physiotherapy. Exclusion criteria: metal } \\
\text { prosthesis in some part of the body; cardiac pacemaker; either cognitive impairment, psychotic, or either schizophrenic } \\
\text { disorders; neuropsychiatric co-morbidity; drugs that reduce seizure threshold or spasticity }\end{array}$ \\
\hline \multirow[t]{4}{*}{ Study type } & Interventional allocation: randomized \\
\hline & Masking: double-blind \\
\hline & Assignment: crossover \\
\hline & Primary purpose: treatment \\
\hline Date of first enrolment & December 2017 \\
\hline Target sample size & 20 \\
\hline Recruitment status & Recruiting \\
\hline Primary outcome(s) & Change in motor scores from baseline to four weeks \\
\hline $\begin{array}{l}\text { Key secondary } \\
\text { outcome(s) }\end{array}$ & Assessment of change in sensory and spasticity scores from baseline to four weeks \\
\hline
\end{tabular}

NA not available

codes and will be concealed from the allocation process by one different researcher. The researchers responsible for applying the intervention and the outcome assessors will not know the study design, allocation, objectives, and expected outcomes.

In addition, for the blinding of the experimenter (responsible for applying the intervention), one assessor (responsible for randomization) will be in charge of giving the active or sham coil to the experimenter. The sham coil has exactly the same shape of the active coil. Further details are presented in the "rTMS-sham" section.

\section{Allocation concealment}

Allocation concealment will successfully be reached since no one involved in this study (i.e. the participants, researchers, and outcome assessors) will be aware of the treatment allocations. Furthermore, investigators will have no control over the order of patients randomized. A blinded investigator will encode the individuals and groups of intervention. To perform the allocation procedure, the encoded groups will be placed inside a closed opaque envelope, which will be labeled with the code for each participant. Envelopes will be opened only during the time of active or sham intervention. 


\section{Intervention}

All participants will attend the assigned rTMS intervention as follows: there will be ten sessions over two weeks, in which five sessions will be active and five will be sham, separated by a two-week washout period. The sessions will be administered consecutively and once a day. The researchers will be trained to perform both rTMS-sham and rTMS-active interventions.

\section{rTMS-active}

Over five consecutive sessions per week (i.e. one session daily), rTMS-active will be performed with a frequency of $5 \mathrm{~Hz}$ and 12 pulse trains. The stimulation intensity will be set at $100 \%$ of the motor threshold of the area corresponding to the abductor pollicis brevis. The stimulation target will be the area associated with the bilateral lower-limb motor area (i.e. vertex, Cz) of the M1.

We chose an intensity of $100 \%$, based upon the limitations reported by Kumru et al. [16], who showed that an intensity of $90 \%$ applied during active rTMS was relatively low for leg muscles. In this line of reasoning, Rossini et al. [17] suggests that stronger descending excitatory drive shall be obtained by higher stimulus intensities, thereby yielding a faster temporal-spatial summation on corticomotoneuron connections. We have also taken into consideration that some studies suggest the use of even higher intensities (some used 110-120\% in individuals with Parkinson's disease, for example) considering the elevated motor threshold of lower-limb muscles [33, 34]. The intensity of $100 \%$ was then chosen according the safety criteria suggested by Rossi et al. [9].

\section{rTMS-sham}

The rTMS-sham will be performed over five consecutive sessions per week (one session daily.) The sham coil will be used because it ensures the attenuation of the magnetic field while appearing to be the same shape as the active coil, with good approximation of auditory feedback [35]. In addition, the tactile contact of the coil with the skull is maintained.

\section{Procedures}

The participants will be positioned comfortably in either a normal chair or a wheelchair, depending on the level of motor commitment of each patient. The feet will be positioned flat on the floor and the hands will rest on the thigh, in the supine position.

The rTMS stimulator will be connected to a figureeight coil (i.e. butterfly coil) and positioned on the vertex of the lower-limb motor area, which corresponds to the apical surface of the skull. We chose to stimulate the vertex point because the motor impairment of subjects with iSCI occurs bilaterally, although the degree of involvement varies from one side to the other. Therefore, both cortical sides will be stimulated simultaneously, as the vertex point is equidistant between the left and right hemispheres [36, 37]. Standardized caps will be used, in accordance with the International 10-20 system EEG. Such a procedure will be used to find the motor threshold, a point which corresponds to the motor area of the abductor pollicis brevis, and so marks the point corresponding to the vertex.

\section{rTMS protocol \\ Magnetic stimulator}

The rTMS will be applied using Neurosoft - Neuro-MS 5 (Neurosoft Ltd $^{\oplus}$, Ivanovo, Russia), a commercially available transcranial magnetic stimulator equipped with an angulated figure-eight-shaped coil (AFEC - 01-100).

\section{Target}

rTMS will be applied to an angulated figure-eight coil over the lower-limb motor area localized in M1 (in order to stimulate both lower limbs), with the handle of the coil parallel to the interhemispheric midline (pointing occipitally) as used by Khedr et al. [33], Jetté et al. [38], and Ji et al. [39] based on the vertex position of the International 10-20 system EEG.

When the orientation of the handle of a figure-eight coil is parallel to the interhemispheric midline (posterior-anterior direction), there is a TMS motor cortex activation through the preferential recruitment of cortical interneurons and through activating the pyramidal tract indirectly $[17,34,40,41]$. Thus, the biological effect depends on the neuronal circuit finally recruited [17].

\section{rTMS sessions}

Each session will be held at the Neuromodulation Laboratory of UEPB, using the same equipment during the same time of day. Each session will consist of 12 trains of 50 magnetic pulses at $5 \mathrm{~Hz}$ on each train. These pulses will be separated by 10-s intervals between each train. Intensity will be set at $100 \%$ of the individual's RMT, defined as the lowest stimulation intensity that, within ten trials, induced at least five motor-evoked potentials assessed on the first dorsal interosseous muscle in the resting state. The parameters of rTMS were chosen based on safety parameters of the both National Institute of Neurological Disorders and Stroke (NINDS) and the paper by Rossi et al. [9].

\section{Primary outcomes}

To evaluate the motor and sensory effects of the highfrequency rTMS in the lower-limb motor area on M1 (i.e. vertex, $\mathrm{Cz}$ ) and check the possibility of generating motor gains in participants with iSCI, we will observe the change from baseline motor values provided by the ASIA score. 
Only one researcher will be responsible for evaluating all of the participants through both the sensorimotor scales and electromyography (EMG). This researcher will be a physiotherapist who will also receive training to use the scales and EMG device. In addition, we will perform an evaluation of the intra-rater reliability of the instruments used in this study. Adverse events will be collected using a form for adverse events at each assessment point and any adverse event related to the intervention will be reported.

\section{ASIA - motor score}

The International Standards for Neurological Classification of Spinal Cord Injury (ISNCSI) is a medical examination from which part of the ASIA motor score is derived [42]. It uses a test of the strength of ten key muscles on each side of the body (e.g. elbow flexors, wrist extensors, hip flexors, quadriceps, dorsi flexors). The score ranges from 0 (no contraction) to 5 (normal resistance) through a full range of motion. A total possible score of 50 for the upper extremities (UE) and 50 for the lower extremities (LE) may be obtained [42].

\section{Secondary outcomes}

ASIA - sensory score

The ASIA sensory score is also part of the assessment for the ISNCSI [42]. The test involves pinprick and light touch sensation at key points representing each dermatome of the body, scored on a three-point scale $(0,1$, and 2$)$. Scores will be summed to give a total possible score of 224, where a higher score indicates better sensation than a lower score [42].

\section{Fugl-Meyer scale for upper and lower members (motor part} - FMS)

The FMS is an instrument used to evaluate body function impairment after stroke [43]. This scale assesses five domains: motor; sensory; balance; range of motion; and joint pain [43]. The UE (i.e. shoulder, elbow, forearm, wrist, and hand) and LE (i.e. hip, knee, and ankle) are assessed within the motor domain, then impairment severity and functional ability are indexed [44]. Sullivan et al. [44] affirm that it is likely that the FMS motor score may be a clinical measure indicative of white matter damage within CST fibers. This potential can justify its use in SCI populations. This scale is a 226-point multi-item Likert-type scale used to measure recovery. Multiple items are scored on a three-point ordinal scale $(0=$ cannot perform, $1=$ perform partially, $2=$ performs fully) [43]. In addition, the motor domain includes score ranges from 0 (plegia) to a maximum of 100 (normal motor performance); 66 points can be divided for the UE and 34 points for the $\operatorname{LE}[44,45]$. In this study we will only use the motor domain and evaluation will take place bilaterally.

\section{Surface electromyography (EMG)}

The EMG will be recorded differentially using roundshaped surface electrodes $(\mathrm{Ag}-\mathrm{AgCl}, 0.8 \mathrm{~cm}$ diameter, with an inter-electrode distance of $2 \mathrm{~cm}$ ) over the following muscles: vastus medialis; vastus lateralis; rectus femoris; biceps femoris; gastrocnemius; and tibialis anterior. We will position the electrodes at standard Surface ElectroMyoGraphy for the Non-Invasive Assessment of Muscles (SENIAM) positions [46], and a ground electrode will be over the tibia of the left leg. The EMG signals will be amplified and filtered ( $5 \mathrm{~Hz}$ to $2 \mathrm{kHz}$ ) by a biological data acquisition system Miotool 400 (Miotec ${ }^{\circ}$, Brazil) and sent to an A/D interface (National Instruments, Austin, TX, USA) with a 2-kHz sampling rate. Data will be stored on hard disk for later off-line processing. Data will be collected using the software Miograph $\left(\right.$ Miotec $\left.^{\circ}\right)$. The EMG acquisitions will be obtained during knee extension, knee flexion, plantar flexion, and dorsiflexion. For $1 \mathrm{~min}$, participants will perform contractile movements with speed controlled by audible and visual signals. The normalization procedure for peak contraction will be made to standardize EMG acquisitions and make them comparable.

\section{Modified Ashworth Scale (MAS)}

The MAS is an instrument used to evaluate the spasticity (i.e. grading the resistance encountered during such passive muscle stretching) $[47,48]$. The grades of spasticity are 0 (normal muscle tone), 1 (slight increase in muscle tone, when move a limb), 2 (more marked increase in muscle tone, but limb easily flexed), 3 (considerable increase in muscle tone), and 4 (limb rigid in flexion or extension) [47].

\section{Socio-demographic questionnaire (SDQ)}

The SDQ will be used to collect personal, socioenvironmental, and clinical information. It will also determine information used to evaluate participants' eligibility criteria. Our research center standardized this qualitative instrument, but a pilot test will be conducted to identify possible necessary adjustments to this questionnaire. In summary, this scale will be used as a sample description and as an eligibility criterion.

\section{Scale of Mini-Mental State Examination}

The MMSE assesses the mental state of the individuals assessed $[49,50]$. The maximum score is 30 and there are two domains. The first tests for vocal responses, which covers orientation, memory, and attention (maximum of 21 points). The second tests for the ability to name objects, obey verbal and written commands, 
write a sentence spontaneously, and copy a complex polygon (maximum of nine points) [50, 51]. In Brazil, Bertolucci et al. [52] translated the MMSE. They found that the educational level of participants influenced their total score on the MMSE. As a result, the authors have proposed different cut-offs for the diagnosis of cognitive decline. The suggested cutoff points were 13 for illiterates, 18 for low and middle school, and 26 for high school. We will use this scale as an eligibility criterion.

\section{Hamilton Rating Scale for Depression}

The HAM-D is used to identify depression and contains 17 items plus four additional variables (diurnal variation, derealization, paranoid symptoms, and obsessive symptoms) [53]. Hamilton has not established a cut-off point, but in clinical practice scores higher than 25 points characterize severely depressed patients; scores in the range of 18-24 indicate moderate depression; and scores in the range of 7-17 indicate mild depression [54]. We will use this scale as an eligibility criterion.

\section{Electroencephalogram}

EEG provides a measurement of brain electrical activity (i.e. the amount of synaptic activity synchronized) recorded from scalp electrodes [55]. Applications of EEG can be used in identification of nature, type, and severity of epileptiform activity [56]. In this study, the EEG will be used to identify individuals with epilepsy or brainactivity patterns that may indicate possible underlying seizures. Therefore, this exam will serve only as an eligibility criterion.

\section{Neurological examination (NE)}

Clinical data collection will be achieved via qualitative standardized NE. This instrument consists of four parts: a history of the lesion and clinical symptoms; sensory and motor evaluation; functional activities; superficial and deep reflections.

\section{Statistical analysis \\ Sample size}

The sample size was calculated using statistical software (GPower 3.1.5) [57] on the main outcome measure (i.e. the motor score). This calculation was based on data from one study with a group of SCI individuals that received high-frequency rTMS at a frequency of $5 \mathrm{~Hz}$ on the vertex, which related to an improvement in clinical measures of the motor score [24]. The power was 0.80 ; the alpha was 0.05 , the effect size was 0.65 (Cohen's $d$ ). The sample estimation indicated that 14 participants would be necessary (i.e. seven per group). With an adjustment to allow for a dropout rate, we will recruit 20 participants.

\section{Data analysis}

The analysis will follow a pre-specified analysis plan, based on comparing the groups as randomized (intention-totreat). All data will be presented as mean \pm standard deviation and summarized in frequency tables. We will use Microsoft Office Excel for Windows (version 2013) to store the data and the Statistical Package for Social Sciences (SPSS-IBM ${ }^{\circ}$ ) version 21 for Windows to perform statistical analysis. The normality and homogeneity of all variables (i.e. primary and secondary outcomes) will be tested with the Shapiro-Wilk and Levene's test, respectively. For non-parametric data, the Friedman test will be used followed by the Wilcoxon post hoc test. We will use the ANOVA two-way $(2 \times 4)$ for the inferential statistic of the parametric data, with Tukey's post hoc tests. Significance level will be set as $\alpha \leq 0.05$ and the effect size will be calculated by eta-squared $\left(\eta^{2}\right)$.

\section{Expected risks}

High-frequency rTMS is a safe non-invasive technique according to current knowledge $[9,58]$. Some studies indicate that there may be weak tension headaches and muscle spasms experienced during rTMS [59]. Overall, in cases where contraindications or safety parameters were not maintained, serious adverse events such as seizures occurred [9]. According to Rossi et al. [9], a large number of individuals were subjected to studies with rTMS since 1998 (when the limit parameters by Wassermann et al. [58] were defined) and a small number of seizures were observed. Therefore, the risk of rTMSinduced seizures is considered very low. To avoid adverse effects and risks to the individual, the rTMS parameters used in the present trial will be within the safety limits set by the NINDS and by Rossi et al. [9], which were updated security settings for transcranial magnetic stimulation.

\section{Expected benefits}

Intervention with high-frequency rTMS over the parameters established in this study may generate motor gains and consequent functional recovery in patients with iSCI. Motor gains and functional recovery might contribute to an improved quality of life and functional independence. Moreover, the participation of those in this study will contribute towards the construction of scientific knowledge on the use of rTMS in iSCI.

\section{Discussion}

Individuals with iSCI show clinical symptoms which are associated with reduced quality of life and functional independence $[60,61]$. The CNS may be able to recover naturally after injury due to plasticity mechanisms and reorganization of residual neural pathways [5]. However, natural recovery is limited and cannot be relied upon 
[6]. It is therefore plausible that stimulation as a rehabilitation technique may be crucial in enhancing CNS plasticity. High-frequency rTMS has emerged as a promising technique in stimulating neural reorganization and synaptic plasticity in cortical and subcortical networks, thereby affecting the descending control of spinal excitability [11, 12, 14, 34, 62, 63]. These mechanisms might accelerate the development of neural connectivity responsible for motor function improvement [12, 14, 22, 24, 63].

Although an adaptive reorganization involves the formation of new connections and restoration of preexisting connections, studies that directly address quantitative parameters associated with neuronal repair are limited. This is because most of the outcome measures are not able to assess the neurophysiological substrates that directly contribute to functional recovery, such as specific mechanisms within the brain and spinal cord that influence the generation of the motor command to the affected limbs [63].

The protocol described herein is expected to be the first fully randomized controlled double-blind crossover trial to assess the effect sizes associated with rTMS intervention in participants with iSCI. We expect the outcomes of the present study to provide additional clinical evidence of the potential benefits of high-frequency rTMS applied to the lower-limb motor area to improve sensorimotor recovery and/or to reduce spasticity in patients with iSCI.

\section{Trial status}

Participant recruitment started in May 2017 and is expected to end in November 2017. Study completion is estimated by May 2018.

\section{Additional file}

Additional file 1: SPIRIT 2013 Checklist. rTMS study protocol. (DOC $122 \mathrm{~kb}$ )

\begin{abstract}
Abbreviations
ASIA: American Spinal Injury Association Impairment Scale; CAEE: Certificado de Apresentação para Apreciação Ética (in Portuguese); CAPES: Coordination for Higher Education Staff Development (in Portuguese); CNS: Central nervous system; CST: Corticospinal tract; EEG: Electroencephalogram; EMG: Electromyography; FAPESP: Foundation of Support for Research of São Paulo State (in Portuguese); FMS: Fugl-Meyer Scale; HAM-D: Hamilton Rating Scale for Depression; iSCI: Incomplete spinal cord injury; ISNCSI: International Standards for Neurological Classification of Spinal Cord Injury; LE: Lower extremity; M1: Primary motor cortex; MAS: Modified Ashworth Scale; MMSE: Mini-Mental State Examination; NE: Neurological examination; NINDS: National Institute of Neurological Disorders and Stroke; rTMS: Repetitive transcranial magnetic stimulation; SCI: Spinal cord injury; SDQ: Socio-demographic questionnaire; SENIAM: Surface Electromyography for the Non-Invasive Assessment of Muscles; SPIRIT: Standard Protocol Items: Recommendations for Interventional Trials; SPSS: Statistical Package for Social Sciences; UE: Upper extremity
\end{abstract}

\section{Acknowledgments}

The authors thank Paraíba State University for the availability of the Laboratory of Neuroscience and Behavior and University of São Paulo for the support to this study. The last author (FHM) would like to thank FAPESP (grant \#2015/ 13096-1) for supporting his research and for covering the publication costs of the present paper. The first author (AVLA) is supported by the Staff development coordination of superior education in Brazil (Coordenação e Aperfeiçoamento de Pessoal de Ensino Superior - CAPES).

\section{Funding}

The last author (FHM) is currently supported by a research grant from FAPESP \#2015/13096-1 (Foundation of Support for Research of São Paulo State), which covered the publication costs of this paper.The fisrt author (AVLA) holds a scholarship from a government agency in Brazil, in charge of promoting high standards for postgraduate courses (Coordination for Higher Education Staff Development - CAPES). The financial sponsors will play no role in the design, execution, analysis and interpretation of data, or writing of the study.

\section{Availability of data and materials}

The datasets generated and analyzed during the current study are not publicly available because the protocol has not been completed at the time of submission (see "Trial status" above), but will be available from the corresponding author on reasonable request.

\section{Authors' contributions}

AVLA, VRNB, GSG, CBMM, and FHM conceived and designed the study. These authors plus TM, TDS, DOS, SLF, JT, and FF participated in drafting the article and reviewed critically for important intellectual content. All authors have read and approved the final version of the manuscript.

\section{Ethics approval and consent to participate}

This research project was approved by the Ethical Committee of Paraiba State University, under the number CAEE: 18753713.0.0000.5187. Informed consent will be obtained by main researcher from each participant or his legal guardian before data collection starts. We will conduct this study under the rules of Resolution 466/12 [64] and Declaration of Helsinki $[65,66]$. Data will be stored electronically in a database, with secure and restricted access. Data transfer will be encrypted and any information capable of identifying individuals removed. The authors plan to disseminate the findings of the study in peer-reviewed journals and at relevant scientific conferences.

Consent for publication

Not applicable

\section{Competing interests}

The authors declare that they have no competing interests.

\section{Publisher's Note}

Springer Nature remains neutral with regard to jurisdictional claims in published maps and institutional affiliations.

\section{Author details}

${ }^{1}$ School of Arts, Sciences and Humanities, EACH - USP, University of São Paulo, São Paulo, Brazil. ${ }^{2}$ Department of Physiotherapy - UEPB, Paraíba State University, Campina Grande, Brazil. ${ }^{3}$ Harvard Center for Noninvasive Brain Stimulation, Harvard Medical School, Boston, MA, USA. ${ }^{4}$ Faculty of Medicine, University of British Columbia, Vancouver, BC, Canada. ${ }^{5}$ Physical Therapy Program - UNESP, State University of São Paulo, São Paulo, Brazil. ' University of Exeter Medical School, Exeter, UK. ${ }^{7}$ University of Lincoln, Lincoln, UK. ${ }^{8}$ Haven Clinical Psychology Practice, Cornwall, UK. ${ }^{9}$ Biomedical Engineering Laboratory, Department of Telecommunication and Control, Avenida Professor Luciano Gualberto, Travessa 3, n. 158. Cidade Universitária, São Paulo, SP 05508-010, Brazil.

Received: 5 May 2017 Accepted: 25 October 2017

Published online: 06 November 2017

\section{References}

1. Calabró RS, Naron A, Leo A, Bramanti P. Usefulness of robotic gait training plus neuromodulation in chronic spinal cord injury: a case report. J Spinal Cord Med. 2017:40(1):118-21.

2. Fechio MB, Pacheco KMB, Kaihami HN, Alves VLR. A repercussão da lesão medular na identidade do sujeito. Acta Fisiátrica. 2009;16:38-42. 
3. Hill MR, Noonan VK, Sakakibara BM, Miller WC. SCIRE Research Team. Quality of life instruments and definitions in individuals with spinal cord injury: a systematic review. Spinal Cord. 2009;48(6):438-50.

4. Herrmann KH, Kirchberger I, Biering-Sorensen F, Cieza A. Differences in functioning of individuals with tetraplegia and paraplegia according to the International Classification of Functioning, Disability and Health (ICF). Spinal Cord. 2011;49(4):534-43.

5. Raineteau O, Schwab ME. Plasticity of motor systems after incomplete spinal cord injury. Nat Rev Neurosci. 2001;2(4):263-73.

6. Fawcett JW, Curt A, Steeves JD, Coleman WP, Tuszynski MH, Lammertse D, et al. Guidelines for the conduct of clinical trials for spinal cord injury as developed by the ICCP panel: spontaneous recovery after spinal cord injury and statistical power needed for therapeutic clinical trials. Spinal Cord. 2007; 45(3):190-205

7. Oudega M, Perez MA. Corticospinal reorganization after spinal cord injury. J Physiol. 2012;590(16):3647-63.

8. Kobayashi M, Pascual-Leone A. Transcranial magnetic stimulation in neurology. Lancet Neurol. 2003;2(3):145-56.

9. Rossi S, Hallett M, Rossini PM, Pascual-Leone A. Safety of TMS Consensus Group. Safety, ethical considerations, and application guidelines for the use of transcranial magnetic stimulation in clinical practice and research. Clin Neurophysiol. 2009;120(12):2008-39.

10. Tazoe T, Perez MA. Effects of repetitive transcranial magnetic stimulation on recovery of function after spinal cord injury. Arch Phys Med Rehabil. 2015;96 Suppl 4:145-55.

11. Ridding MC, Rothwell JC. Is there a future for therapeutic use of transcranial magnetic stimulation? Nat Rev Neurosci. 2007;8(7):559-67.

12. Bunday KL, Perez MA. Motor recovery after spinal cord injury enhanced by strengthening corticospinal synaptic transmission. Curr Biol. 2012;22(24):2355-61.

13. Kumru H, Murillo N, Samso JV, Valls-Sole J, Edwards D, Pelayo R, et al. Reduction of spasticity with repetitive transcranial magnetic stimulation in patients with spinal cord injury. Neurorehabil Neural Repair. 2010;24(5): 4335-441.

14. Benito J, Kumru H, Murillo N, Costa U, Medina J, Tormos JM, et al. Motor and gait improvement in patients with incomplete spinal cord injury induced by high-frequency repetitive transcranial magnetic stimulation. Top Spinal Cord Inj Rehabil. 2012;18(2):106-12.

15. Belci M, Catley M, Husain M, Frankel HL, Davey NJ. Magnetic brain stimulation can improve clinical outcome in incomplete spinal cord injured patients. Spinal Cord. 2004:42:417-9.

16. Kumru H, Benito-Penalva J, Valls-Sole J, Murillo N, Tormos JM, Flores C, et al. Placebo-controlled study of rTMS combined with Lokomat ${ }^{\oplus}$ gait training for treatment in subjects with motor incomplete spinal cord injury. Exp Brain Res. 2016;234(12):3447-55.

17. Rossini PM, Burke D, Chen R, Cohen LG, Daskalakis Z, Di lorio R, et al. Noninvasive electrical and magnetic stimulation of the brain, spinal cord, roots and peripheral nerves: Basic principles and procedures for routine clinical and research application. An updated report from an I.F.C.N. Committee. Clin Neurophysiology. 2015;126(6):1071-107.

18. Gunduz A, Kumru H, Pascual-Leone A. Outcomes in spasticity after repetitive transcranial magnetic and transcranial direct current stimulations. Neural Regen Res. 2014;9(7):712-8.

19. Gentner R, Wankerl K, Reinsberger C, Zeller D, Classen J. Depression of human corticospinal excitability induced by magnetic theta-burst stimulation: evidence of rapid polarity-reversing metaplasticity. Cereb Cortex. 2008;18(9):2046-53.

20. lezzi E, Conte A, Suppa A, Agostino R, Dinapoli L, Scontrini A, et al. Phasic voluntary movements reverse the aftereffects of subsequent theta-burst stimulation in humans. J Neurophysiol. 2008;100(4):2070-6.

21. Ridding MC, Ziemann U. Determinants of the induction of cortical plasticity by non-invasive brain stimulation in healthy subjects. J Physiol. 2010;588(13): 2291-304.

22. Ellaway PH, Vasquez N, Craggs M. Induction of central nervous system plasticity by repetitive transcranial magnetic stimulation to promote sensorimotor recovery in incomplete spinal cord injury. Front Integr Neurosci. 2014;8:12

23. Nardone R, Holler $Y$, Thomschewski A, Brigo F, Orioli A, Holler P, et al. rTMS modulates reciprocal inhibition in patients with traumatic spinal cord injury. Spinal Cord. 2014;52(11):831-5.

24. Kuppuswamy A, Balasubramian AV, Maksimovic R, Mathias CJ, Gall A, Craggs $M D$, et al. Action of $5 \mathrm{~Hz}$ repetitive transcranial magnetic stimulation on sensory, motor and autonomic function in human spinal cord injury. Clin Neurophysiol. 2011;122(12):2452-61.

25. Lammertse D, Tuszynski MH, Steeves JD, Curt A, Fawcett JW, Rask C, et al. Guidelines for the conduct of clinical trials for spinal cord injury as developed by the ICCP panel: clinical trial design. Spinal Cord. 2007;45(3): 232-42.

26. Chan AW, Tetzlaff JM, Gotzsche PC, Altman DG, Mann H, Berlin JA, et al. SPIRIT 2013 explanation and elaboration: guidance for protocols of clinical trials. BMJ. 2013;346:e7586.

27. Baeken $C L$, Vanderhasselt MA, Remue J, Herremans S, Vanderbruggen $N$, Zeeuws $\mathrm{D}$, et al. Intensive HF-rTMS treatment in refractory medicationresistant unipolar depressed patients. J Affect Disord. 2013;151(2):625-31.

28. Lee J, Thumbikat P. Pathophysiology, presentation and management of spinal cord injury. Surgery. 2015;33(6):238-47.

29. MacDonald JW, Sadowsky C. Spinal-cord injury. Lancet. 2002;359(9304):417-25.

30. Monif $\mathrm{M}$, Seneviratne U. Clinical factors associated with the yield of routine outpatient scalp electroencephalograms: A retrospective analysis from a tertiary hospital. J Clin Neurosci. 2017;45:110-14.

31. Smith S. EEG in the diagnosis, classification, and management of patients with epilepsy. J Neurol Neurosurg Psychiatry. 2005;76 Suppl 2:ii2-7.

32. R Development Core Team. R: A language and environment for statistical computing. Vienna, Austria: R Foundation for Statistical Computing; 2010. http://www.R-project.org.

33. Khedr EM, Farweez HM, Islam H. Therapeutic effect of repetitive transcranial magnetic stimulation on motor function in Parkinson's disease patients. Eur J Neurol. 2003;10(5):567-72.

34. Lefaucheur JP, André-Obadia N, Antal A, Ayache SS, Baeken C, Benninger $\mathrm{DH}$, et al. Evidence-based guidelines on the therapeutic use of repetitive transcranial magnetic stimulation (rTMS). Clin Neurophysiol. 2014;125(11): 2150-206.

35. Duecker F, Sack AT. Rethinking the role of sham TMS. Front Psychol. 2015;6:210,

36. Yilmaz B, Kesikburun S, Yasar E, Tan AK. The effect of repetitive transcranial magnetic stimulation on refractory neuropathic pain in spinal cord injury. J Spinal Cord Med. 2014;37(4):397-400.

37. Pitcher D, Garrido L, Walsh V, Duchaine BC. Transcranial magnetic stimulation disrupts the perception and embodiment of facial expressions. J Neurosci. 2008;28(36):8929-33.

38. Jetté F, Côté I, Meziane HB, Mercier C. Effect of single-session repetitive transcranial magnetic stimulation applied over the hand versus leg motor area on pain after spinal cord injury. Neurorehabil Neural Repair. 2013;27(7):636-43.

39. Ji SG, Cha HG, Kim MK. Effects of repetitive transcranial magnetic stimulation on motor recovery in lower extremities of subacute stage incomplete spinal cord injury patients: a randomized controlled trial. J Magnetics. 2015;20(4):427-31.

40. Sakai K, Ugawa Y, Terao Y, Hanajima R, Furubayashi T, Kanazawa I. Preferential activation of different I waves by transcranial magnetic stimulation with a figure-of-eight-shaped coil. Exp Brain Res. 1997;113:24-32.

41. D'Ostilio K, Goetz SM, Hannah R, Ciocca M, Chieffo R, Jui-Cheng A, et al. Effect of coil orientation on strength-duration time constant and l-wave activation with controllable pulse parameter transcranial magnetic stimulation. Clin Neurophysiology. 2016;127(1):675-83.

42. Kirshblum SC, Burns SP, Biering-Sorensen F, Donovan W, Graves DE, Jha A. International standards for neurological classification of spinal cord injury (Revised 2011). J Spinal Cord Med. 2011;34(6):535-46.

43. Fugl-Meyer AR, Jaasko L, Leyman I, Olsson S, Steglind S. The post-stroke hemiplegic patient. 1. a method for evaluation of physical performance. Scand J Rehabil Med. 1975;7(1):13-31.

44. Sullivan KJ, Tilson JK, Cen SY, Rose DK, Hershberg J, Correa A, et al. Fugl-Meyer assessment of sensorimotor function after stroke: standardized training procedure for clinical practice and clinical trials. Stroke. 2011;42(2):427-32.

45. Gladstone DJ, Danells CJ, Black SE. The Fugl-Meyer assessment of motor recovery after stroke: a critical review of its measurement properties. Neurorehabil Neural Repair. 2002;16(3):232-40.

46. Hermes HJ, Freriks B, Disselhorst-Klug C, Rau G. Development of recommendations for SEMG sensors and sensor placement procedures. J Electromyog Kinesiol. 2000;10:361-74.

47. Ashworth B. Preliminary trial of carisoprodal in multiple sclerosis. Practioner. 1964;192:540-2.

48. Ghotbi N, Ansari NN, Naghdi S, Hasson S. Measurement of lower-limb muscle spasticity: Intrarater reliability of Modified Modified Ashworth Scale. J Rehabil Res Dev. 2011;48(1):83-8. 
49. Folstein MF, Folstein SE, Mchugh PR. "Mini-mental state". A practical method for grading the cognitive state of patients for the clinician. J Psychiatr Res. 1975;12(3):189-98.

50. Brucki SMD, Nitrini R, Caramelli P, Bertolucci PHF, Okamoto $H_{H}$. Suggestions for utilization of the mini-mental state examination in Brazil. Arq NeuroPsiquiatr. 2003;61:3B.

51. Cockrell JR, Folstein MF. Mini-Mental State Examination. Princ Pract Geriatric Psych. 2002;2:140-41.

52. Bertolucci PHF, Brucki SMD, Campacci SR, Juliano Y. The Mini-Mental State Examination in an outpatient population: influence of literacy. Arq NeuroPsiquiatr. 1994;52:1.

53. Williams JBW. Standardizing the Hamilton Depression Rating Scale: past, present, and future. Eur Arch Psychiatry Clin Neurosci. 2001;251 suppl 2:I//6-12

54. Gallucci NJ, Campos JMS, Hübner CK. Escala de Depressão de Hamilton (HAM-D): revisão dos 40 anos de sua utilização. Rev Fac Ciênc Méd. 2001; 3(1):10-4.

55. Babiloni C, Pizzella V, Gratta CD, Ferretti A, Romani GL. Fundamentals of electroencefalography, magnetoencefalography, and functional magnetic resonance imaging. Int Rev Neurobiol. 2009;86:67-80.

56. Kannathal N, Choo ML, Acharya UR, Sadasivan PK. Entropies for detection of epilepsy in EEG. Comput Methods Programs Biomed. 2005;80(3):187-94.

57. Faul F, Erdfelder E, Lang AG, Buchner A. G*Power 3: A flexible statistical power analysis program for the social, behavioral, and biomedical sciences. Behav Res Methods. 2007;39:175-91.

58. Wassermann EM. Risk and safety of repetitive transcranial magnetic stimulation: report and suggested guidelines from the International Workshop on the Safety of Repetitive Transcranial Magnetic Stimulation, June 5-7, 1996. Electroencephalogr Clin Neurophysiol. 1998;108(1):1-16.

59. Gomes-Osman J, Field-Fote EC. Improvements in hand function in adults with chronic tetraplegia following a multiday $10-\mathrm{Hz}$ repetitive transcranial magnetic stimulation intervention combined with repetitive task practice. J Neurol Phys Ther. 2015;39(1):23-30.

60. Burr JF, Shephard RJ, Zehr EP. Physical activity after stroke and spinal cord injury: evidence-based recommendations on clearance for physical activity and exercise. Can Fam Physician. 2012:58(11):1236-9.

61. Yang H, Liu CC, Wang CY, Zhang Q, An J, Zhang L, et al. Therapeutical strategies for spinal cord injury and a promising autologous astrocyte-based therapy using efficient reprogramming techniques. Mol Neurobiol. 2016; 53(5):2826-42.

62. Valero-Cabré A, Oliveri M, Gangitano M, Pascual-Leone A. Modulation of spinal cord excitability by subthreshold repetitive transcranial magnetic stimulation of the primary motor cortex in humans. Neuroreport. 2001; 12(17):3845-8.

63. Potter-Baker KA, Janini DP, Frost FS, Chabra P, Varnerin N, Cunningham DA, et al. Reliability of TMS metrics in patients with chronic incomplete spinal cord injury. Spinal Cord. 2016;54(11):980-90.

64. Resolução $n^{\circ}$ 466, de 12 de dezembro de 2012. Ministério da Saúde. http:// bvsms.saude.gov.br/bvs/saudelegis/cns/2013/res0466_12_12_2012.html. Accessed 03 April 2017.

65. Declaration of Helsinki. Somerset West, South Africa: 48th General Assembly-WMA. 6 October 2000. World Medical Association. http://www. wma.net. Accessed 02 April 2017.

66. Declaration of Helsinki. Edinburgh, Scotland: 52nd General Assembly-WMA 13 October 2000. World Medical Association. http://www.wma.net. Accessed 03 April 2017

\section{Submit your next manuscript to BioMed Central and we will help you at every step:}

- We accept pre-submission inquiries

- Our selector tool helps you to find the most relevant journal

- We provide round the clock customer support

- Convenient online submission

- Thorough peer review

- Inclusion in PubMed and all major indexing services

- Maximum visibility for your research

Submit your manuscript at www.biomedcentral.com/submit 